\title{
Las economías del Magreb
}

\section{Pere Mir}

Universitat de Lleida. Pl. Víctor Siurana, s/n. 25003 Lleida. Spain

\section{Resumen}

La situación económica del Magreb es muy dificil. El boom de los hidrocarbutos y el fosfato posibilitó un rápido crecimiento de las industrias básicas, así como de la renta per cápita, pero aplazó la mejora de la agricultura en un contexto de acelerada expansión demográfica. También dio lugar a un elevado nivel de endeudamiento externo, cuya contención ha exigido llevar a cabo políticas de ajuste económico. Tales polfticas, como es bien sabido, conilevan importantes costes sociales.

Palabras clave: Magreb, países subdesarrollados, economía internacional.

\section{Abstract. The Maghreb economies}

The situation of the Maghreb economies is very difficult. The hidtocarbures and phosphate boom stimulated a fast growth of basic industries as well as the income per capita, but it postponed the development of agricultural sector, when the population grew at an accelerated rate. Moreover the level of accumulated external debt forced the adoption of economic adjustment policies, which entailed dranatic social costs.

Key words: Maghreb, Underdeveloped Countries, International Economics.

\section{Sumario}

Introducción La deuda externa y las políticas

Los recursos naturales, de ajuste de los años ochenta

la industrialización

$y$ el comercio exterior

La agricultura y la alimentación

El turismo y las remesas de los emigrantes

Conclustón

Bibliografía 


\section{Introducción}

Durante los últimos 25 años las economías magrebíes han pasado por coyunturas de muy diverso signo ${ }^{1}$. Con el aumento de los precios del crudo y del fosfato en 1974 entraron en una fase expansiva que se acentuó tras los nuevos incrementos de 1979. No obstante, la situación empezó a modificarse ya en 1981, cambio que fue definitivo desde 1986. Con la crisis del Golfo de 1991 se apuntó una nueva coyuntura favorable a los intereses económicos de los países magrebíes. No obstante, dicha alteración no se consolidó. Hoy en día, aunque con matices según el pais, la crisis es muy profunda: la agricultura está atrasada y es incapaz de generar excedentes para poder alimentar a las ciudades mientras que la industria, la cual, centrada en el estadio de primera transformación de los recursos minerales, no ha sabido actuar suficientemente en favor de la expansión del tejido productivo nacional. Con la excepción del marasmo argelino, los ingresos por turismo y las remesas de los emigrantes han pasado a jugar un papel clave en el sostenimiento de la actividad económica, gran parte de la qual se desarrolla dentro de los ambiguos límites del sector informal urbano. Todo ello coincidiendo con un crecimiento demográfico vertiginoso que supera el ritmo de creación de puestos de trabajo - el paro afecta a más del $70 \%$ de la población juvenil-y habiéndose acumulado un volumen desmesurado de deuda externa - cifrado, para el conjunto del Magreb, en más de 56 mil millones de dólares en 1993-.

\section{Los recursos naturales, la industrialización y el comercio exterior}

La extracción de hidrocarburos y de fosfatos para su exportación ha sido uno de los pilares de las economfas magrebíes. La presencia de tales recursos es diferente según el país.

Argelia dispone de importantes reservas de petróleo y, sobre todo, de gas natural. Las primetas exportaciones de crudo coincidieron con los momentos más conflictivos de la guerra de la independencia, ya que no fue hasta finales de 1956 que la empresa francesa Régie Autonome des Pétroles descubrió un yacimiento significativo. La explotación del gas empezó posteriormente: en 1964 se inaguró la primera planta de licuación en Arzew. Este mismo año se fund6 SONATRACH (Société Nationale pour la Recherche, la Production, le Transport, la Transformation et la Commercialisation des Hydrocarbures), empresa que fue absorbiendo las sociedades extranjeras nacionalizadas desde finales de los sesenta ${ }^{2}$.

1. El articulo traca de la evolución económica general de los paises que componen el Magreb geográfico, esto es, Argelia, Marruecos y Túnez. Como es bien sabido, el 27 de febrero de 1989 se firmó en Marraquech el tratado de la Unión del Magreb Árabe que incluye los tres estados cirados más Libia y Mauritania, paises plenamente saharianos. Dicha organización fue activa sobre todo en sus dos primeros ańos.

2. A finales de los ochenta, las reservas de crudo se estimaban en 8,4 mil millones de barriles. Esto es, 33 años al ritmo de extracción de 1989. Por lo que se refiere al gas natural, 
Tabla 1. Composición en (\%) de las exportaciones

\begin{tabular}{lrrrrr}
\hline & 1971 & 1974 & 1980 & 1986 & 1991 \\
\cline { 2 - 6 } Argelia & & & & & \\
Productos agrarios y alimenticios & 13 & 4 & $<1$ & $<1$ & 1 \\
Crudo y GNL & 78 & 88 & 90 & 74 & 77 \\
Productos químicos & 2 & 6 & 9 & 25 & 20 \\
Manufacturas & 7 & 2 & $<1$ & $<1$ & 2 \\
Marruecos & & & & & \\
Productos agrarios y alimenticios & 52 & 26 & 30 & 31 & 30 \\
Fosfatos y minerales & 30 & 60 & 39 & 20 & 10 \\
Productos textiles & 8 & 6 & 11 & 20 & 26 \\
Productos químicos & 4 & 4 & 15 & 21 & 23 \\
Orras manufacturas & 6 & 4 & 5 & 8 & 11 \\
Túnez & & & & & \\
Productos agrarios y alimenticios & 37 & 26 & 7 & 12 & 15 \\
Crudo y minerales & 40 & 48 & 53 & 25 & 13 \\
Productos textiles & 3 & 5 & 19 & 30 & 39 \\
Productos químicos & 12 & 14 & 5 & 22 & 17 \\
Otras manufacturas & 8 & 7 & 16 & 11 & 16 \\
\hline
\end{tabular}

Túnez encontró petróleo muy cerca de la frontera argelina y en un campo off-shore enfrente de las islas Kerkenah. Dado que las reservas del país son escasas, hoy día casi ya no se exporta crudo de origen tunecino (Toumi, 1990). Por otro lado, las prospecciones hechas en Marruecos nunca han tenido éxito.

El fosfato es el principal recurso del reino alauita: concentra la mitad de las reservas mundiales totales, estimadas en 46 mil millones de toneladas. Entre sus inmensos yacimientos sobresale el de Khourigba, donde los estratos con dicho mineral cubren un área de $4.000 \mathrm{~km}^{2}$. Sus poca profundidad y disposición horizontal permiten la extracción a cielo abierto. A pesar de que los USA y la ex-URSS son los principales productores, su elevado consumo interno convierte a Marruecos en el primer exportador: una tercera parte del comercio mundial. La actividad fosfatera marroquí se inició en 1922. La empresa encargada de la extracción, el transporte y la exportación del fosfato y sus derivados es la estatal OCP (Office (Chérifien des Phosphates).

Estos recursos minerales han tenido un peso importante en las exportaciones magrebies ${ }^{3}$ (véase la tabla 1 ).

Argelia es casi exclusivamente exportadora de hidrocarburos, ya que al crudo y al gas natural licuado deben añadirse los productos petroquímicos. El espacio que van ganando éstos últimos se explica por las grandes inversio-

el pais ocupa el cuarto lugar en el ranking mundial, con unas reservas evaluadas en 2,9 billones de $\mathrm{m}^{3}$.

3. Información procedente de fuentes estadísticas internacionales y elaboradas en MIR, 1993. 
nes industriales, especialmente en el subsector petroquímico, llevadas a cabo desde mediados de los setenta ${ }^{4}$. Ello provocó que el número de trabajadores en dicho subsector pasase de 12 mil ocupados en 1969 a más de 93 mil en 1978 (Bennoune, 1988). Durante esta etapa, los bienes de equipo representaron un $40 \%$ del valor de las importaciones. Buena parte de esta industrialización acelerada fue financiada con préstamos exteriores (Goumeziane, 1994).

En el caso de Marruecos, los productos agrarios suelen ocupar la primera plaza en la composición de las exportaciones, excepto en años muy concretos tales como 1974 y 1980. La razón, como se demostrará más adelante, son los altibajos del precio del fosfato. Otra categoría que va progresivamente creciendo es la de los productos químicos que, también para el reino cherifiano, refleja la opción de valorización de los recursos minerales decidida durante la segunda mitad de los años setenta. Se levantaron, pues, grandes complejos químicos para la transformación del fosfato en bruto en ácido fosfórico y abo$\operatorname{nos}^{5}$.

Los artículos de la confección son el otro gran capítulo industrial. Su dinámica expansiva responde a las disposiciones que, en Marruecos desde 1984 y 1985 (Calatrava, 1991) y en Túnez a través de los códigos de inversiones extranjeras de 1972, 1974 y 1987 (véanse ABC, 1986 y Delmasaure, 1990), han otorgado todo tipo de facilidades a las empresas de tráfico de perfeccionamiento. Destaca el caso de Túnez, donde estos productos sólo representaban, a principios de los años setenta, el 3\% del valor exportado, mientras que en 1991 su proporción se acercaba al $40 \%$. Una evolución resultado de la instalación de unas 200 empresas de origen alemán, 180 francés, 40 belga, etc. (Baiget, 1991). El carácter simultáneamente importador de este tipo de industrias queda reflejado en el hecho que, dentro de las compras de productos básicos y semielaborados para la industria - un $45 \%$ de las importaciones totales de Túnez a finales de los años ochenta-, dos terceras partes correspondieron a fibras, cueros y tejidos. A pesar de lo dicho, el Magreb no ocupa un lugar destacado en el comercio mundial de artículos de la confección ${ }^{6}$.

Una vez comprobado-que las exportaciones están muy poco diversificadas y que el grueso de los ingresos recae sobre las materias primas y sus derivados, debe constatarse el elevado grado de apertura al exterior de las economías

4. Así, la formación bruta de capiral fijo respecto al PIB pasó de una media antral del $11,5 \%$ durante el primer quinquenio de los años setenta al $39 \%$ durante el segundo. El valor máximo correspondí al afro 1978, con un extraordinario 51\% deI PIB.

5. Por ejemplo, en 1974 de una extracción total estimada en 20,4 millones de toneladas, el $95 \%$ fue exportada en bruto y el $3 \%$ en forma de productos químicos, siendo el resto destinado al consumo interior (Oualalou, 1975), mientras que, en 1988, tn $55 \%$ de las 25 millones de toneladas producidas entre fosfatos y otras suscancias fertikizantes fueron exportadas en bruto, y poco menos del $45 \%$ restante transformadas en ácido fosfórico y abonos (Séréni, 1991).

6. Por ejemplo, si bien la RFA es el primer comprador de este tipo de productos a Túnez, importa cinco veces más de Hong-Kong y cuatro de Turquía que del pás magtebi (Chevalier \& Kessler, 1990). 
magrebíes. En efecto, la proporción de las exportaciones respecto al PIB ha presentado a menudo valores superiores al $20 \%^{7}$. Ello hace sospechar que la evolución de la renta nacional de los países del Magreb resultó considerablemente afectada por las fluctuaciones de las exportaciones. Unas oscilaciones que, siendo originadas por la inestabilidad en los precios de determinadas materias primas, escapaban al control de las autoridades magrebíes. Ello provocó graves transtornos económicos cuando resultó que las previsiones de ingresos no se cumplían. Esto es: había que tomar una decision arriesgada entre, o bioen frenar los proyectos de inversión en marcha, desechando así los recursos ya utilizados, o bien endeudarse confiando de que cambiase la coyuntura. Un dilema mucho más temible si, como sucedió en el caso magrebí, durante breves periodos las exportaciones de los hidrocarburos y del fosfato se beneficiaron de precios internacionales muy favorables. Veámoslo.

La evolución de las ventas exteriores del Magreb se explica, en buena parte, a partir de las fluctuaciones de los precios del petróleo y de los fosfatos. Con respecto al crudo, como es bien sabido, durante décadas presentó precios bajos y estancados. Empero, el primer trimestre de 1974 el barril se cotizo a 12,4 \$, mientras que un año antes lo había hecho a $2,7 \$$. Cinco años más tarde, se produjo un segundo aumento tepentino del precio del petróleo: de $19,3 \$$ el barril en el segundo trimestre de 1979 a $35,4 \$$ un año más tarde y hasta un máximo del $40,8 \$$ en 1981. Estos elevados precios justificaron la reapertura de campos antes clausurados por el hecho de no ser rentables y animaron la actividad de prospección y explotación donde, por alguna razón, las inversiones previas necesarias eran muy altas. La consiguiente pérdida de peso de la OPEP dentro del mercado mundial y los desacuerdos en su seno, ejemplarizados por el conflicto Irak-Irán, por un lado, y las políticas de ahorro energético y la recesión económica que provocaron las medidas antiinflacionistas de los gobiernos de la OCDE, por el otro, provocaron el desplome de los precios a mediados de los ochenta ${ }^{8}$. Finalmente, en el reciente conflicto del Golfo durante la segunda mitad de 1991, los precios del crudo tuvieron un breve repunte.

El caso del fosfato es, también, muy singular. En 1971 se creó la Phosrock (Phosphate Rock Export Asociation) cártel que agrupaba las principales empresas norteamericanas extractivas y exportadoras de fosfato y derivados. Esta asociación más la OCP marroquí pasaron a ocupar la posición dominante en el mercado mundial: aproximadamente, durante la primera mitad de los ochenta, supuso el $35 \%$ de la extracción total y el $60 \%$ de los intercambios internacionales.

Repentinamente, los precios del fosfato pasaron de los $13 \$$ la tonelada en 1973 a $42 \$$ el primer semestre de 1974 y se alcanzaron los $68 \$$ durante la segunda mitad de este mismo año. Un valor máximo que se mantuvo todo

7. Una cifra elevada si se ciene en cuenta que esce ratio, el año 1982, para países como el Japón, los EE.UU., México y la India era del 3,4\%, 7\%, 12,3\% y 5,6\% respectivamente (véase Mir, 1993).

8. En concreto, $12,2 \$$ el barril en el segundo trimestre de 1986 frente a 30,1 $\$$ un año antes. 
el año siguiente' . Pero en 1976 la media anual de los precios cayó por debajo de los $40 \$$, cifta que tendió hacia los $30 \$$ en el año 78 . No obstante, a partir de entonces los precios del fosfato volvieron a crecer, alcanzándose los $49,5 \$$ en 1981 . Y, de nuevo, otro descenso, de forma que, en 1987 , el precio corriente era el mismo que el de una década antes. Hoy en día la tonelada de fosfato se valora en $33 \$$, tras un leve crecimiento hasta los $42,6 \$$ en 1992.

La punta de mediados de los setenta en la cotización del fosfato fue provocada por la simultaneidad de dos circunstancias:

1. Los grandes productores de cereales tuvieron malas cosechas en los años 1971 y 1972, cosa que provocó una disminución del grano almacenado. Ello generó una presión hacia arriba de los precios agrarios, animando a los agricultores a ampliar la superficie sembrada en los ejercicios siguientes.

2. Además, debido a la primera crisis del petróleo, se habia extendido entre muchos gobiernos una cierta psicosis de futura penuria de materias primas estratégicas, cosa que se tradujo en la creación de estocs de seguridad a cualquier precio.

Ambos factores provocaron una fuerte demanda de abonos que, a su vez, repercutió sobre la de fosfatos. Esto fue aprovechado por la OCP marroquí para, dada su posición dominante en el mercado mundial, triplicar unilateralmente los precios, decisión que fue seguida con satisfacción por el resto de exportadores ${ }^{10}$. No obstante, la subida frenó en un breve plazo el consumo debido a la existencia cercana de sustitutos de los abonos. Además provocó la aparición de nuevos países exportadores, reabriéndose yacimientos antes abandonados. El resultado previsible fue una reducción de los precios de casi a la mitad.

Como era de prever, el efecto de todas estas oscilaciones de precios sobre las exportaciones de las economias del Magreb fue espectacular. Así lo demuestran los siguientes ejemplos:

1. En el caso argelino las ventas exteriores pasaron de 976 millones de dolares en 1971 a 4.943 en 1974 , subiendo a 13.651 en 1980 y bajando hasta 8.065 en 1986.

2. Para Marruecos, las exportaciones subieron de 500 millones de dolares en 1971 hasta 1.704 en 1974 , bajando a 1.530 en el año siguiente.

3. Finalmente, los ingresos de Túnez en 1974 fueron de 872 millones de dolares mientras que en 1981 ascendían a 2.106, descendiendo a 1.763 en 1986. Otro aspecto importante de la descripción del sector exterior de una eco-

9. Debe de ser destacado que, en términos constantes, los $42 \$$ de 1974 eran equivalentes a los $13,4 \$$ de 1952 . Un hecho que llevó a los marroquíes a hablar de actualización de los precios, rechazando que se tratase de una subida abusiva (Oualalou, 1975).

10. La magnitud del aumento parece que fue elegida de forma que contribuyese decididamente a compensat el efecto nocivo sobre la balanza comercial marroquí, tanto del incremento del precio del perróleo como de las compras de armas motivadas por el conflicto del Sahara Occidental (Zenaidi Karray, I987). 
Tabla 2. El peso del comercio con el Magreb (año 1988)

\begin{tabular}{|c|c|c|c|c|c|c|}
\hline & \multicolumn{2}{|c|}{ Francia } & \multicolumn{2}{|c|}{ Italia } & \multicolumn{2}{|c|}{ España } \\
\hline & $\mathrm{X}$ & $\overrightarrow{\mathbf{M}}$ & $\mathrm{X}$ & M & $\bar{X}$ & M \\
\hline Argelia & 0,8 & $\overline{0,9}$ & 0,6 & 1,0 & 0,8 & 0,8 \\
\hline Marruecos & 0,6 & 0,5 & 0,2 & 0,1 & 0,9 & 0,4 \\
\hline Túnez & 0,6 & 0,3 & 0,4 & 0,3 & 0,4 & 0,9 \\
\hline
\end{tabular}

nomía es la distribución territorial de sus socios comerciales. En el caso magrebí, las dos terceras partes de las ventas y compras se llevan a cabo con países de la CEE/UE. La presencia de la Comunidad en las exportaciones magrebíes debe de compararse con el dato siguiente: en 1977, sólo el 2\% del total de importaciones de la CEE/UE procedia del Magreb y, diez años después, la proporción era aún menor: el $1 \%$ (Chevallier \& Kessler, 1990).

Entre los países miembros de la Comunidad, Francia ha sido el mayor partenaire comercial del Magreb en las dos últimas décadas: alrededor del $35 \%$ de las exportaciones (X) y el $\mathbf{4 0 \%}$ de las importaciones $(\mathrm{M})$ argelinas, el $45 \%$ de las X y $50 \%$ de las $M$ marroquíes y, finalmente, casi el $30 \%$ de las $\mathrm{X}$ y el $40 \%$ de las $M$ tunecinas. A continuación, aparecen Italia, la RFA y España. Esta vinculación bilateral ha sido, también, muy asimétrica tal como muestra la tabla 2 referida a los porcentajes de las exportaciones (importaciones) de Francia, Italia y España que fueron destinadas (procedieron) de los tres Estados del Magreb en el año 1988.

Esta asimetría otorga diferente margen de maniobra a los socios en el momento de negociar medida de política comercial. En efecto, a pesar de las ventajas ofrecidas por la CEE/UE a Argelia, Marruecos y Túnez en los sucesivos acuerdos de asociación, la Comunidad ha establecido contingentes, calendarios y otras condiciones restrictivas de importación, especialmente en el caso de los productos agrarios (Pomfret, 1987). Por lo que se refiere a los artículos de la confección, bienes manufacturados en los que los países magrebíes presentan precios muy competitivos, se les ha presionado en ocasiones para que autolimiten sus ventas a la Comunidad (Khader, 1992).

\section{La agricultura y la alimentación}

El potencial agrario del Magreb es muy pequeño. Los datos expuestos en la tabla 3, correspondientes a finales de los ochenta, así lo confirman ${ }^{11}$.

11. Elaboración propia a partir de la confrontación de datos procedentes de Pascon, 1985; Aitt Amara, 1990, y Jordan Galduf, 1991. En general, las fuentes suelen coincidir. La excepción más desracable es la superficie de regadio de Marruecos. Si, por una parte, se cifran en poco más de 700 mil las hectáreas de los grandes perímetros de regadio (Rharb, Moulouya, Tadla, Haouz, Doukkala, erc.), hay mucha divergencia en la estimación de la superficie regada con la denominada pequeña hidráulica (redes de riego locales, pozos, bombas de extracción de agua del subsuelo, etc.). Algunos autores la cuantifican hasca un máximo de 400 mil hectáreas, magnitud que otros rebajan a menos de la mitad. Pot todo ello, el dazo expuesto en la rabla debe de ser entendido como un máximo. 
Sólo el $4 \%$ de la superficie total del Magreb es susceptible de uso agratio. Esto es, poco más de 20 millones de hectáreas, de las cuales menos de una treceaba parte es irrigable en mayor o menor grado. Unas condiciones climáticas, poco o nada favorables al cultivo, explican la exigua proporción de suelo magrebi dedicado a la agricultura. En efecto, el umbral mínimo de los $400 \mathrm{~mm}$ de lluvia necesarios para el crecimiento vegetal sólo se alcanza en las montañas del Atlas y la vertiente atlántica de Marruecos, en una franja paralela a la costa de entre 150 y $200 \mathrm{~km}$ de anchura en Argelia y en la mitad norte de Túnez. Es necesario advertir que esta media antral de lluvias combina largos períodos sin precipitaciones con repentinos e intensos aguaceros torrenciales, los cuales actúan como un poderoso agente de erosión debido a la elevada desforestación del Magreb. En estas condiciones, un retraso en las precipitaciones de otońo dificulta la siembra de los cereales, el cultivo principal. Todas estas razones explican que los rendimientos de las cosechas sean pobres. Por ejemplo, entre 1970 y 1984 , los rendimentos medios de los cereales en Marruecos fueron de $915 \mathrm{~kg} / \mathrm{h}$., con un mínimo de $500 \mathrm{~kg} / \mathrm{h}$. en el año 1981 y un máximo de $1.200 \mathrm{~kg} / \mathrm{h}$. en el año 1976 (Swearingen, 1987). No resulta sorprendente que, en los campos sin riego, sólo una cosecha entre cinco acostumbre a ser satisfactoria, de manera que los rendimientos medios del trigo, siguiendo una rotación bianual, no superan $l o s 600 \mathrm{~kg} / \mathrm{h}$. (Temmar, 1983). Las debilidades de la agricultura explican que, tradicionalmente, la ganadería haya sido un elemento clave para la supervivencia de los campesinos, especialmente el ganado ovino, criado por los pastores nómadas según acuerdos de reciprocidad con los agricultores de la franja costera y los oasis.

La limitada capacidad agraria del Magreb se ha mezclado con problemas de orden socioeconómico. En primer lugar, un patrón muy desigual de distribución de la tierxa y de la asignación de los recursos para la mejora de los rendimientos, especialmente mediante el regadío, en los casos de Marruecos y Túnez y, en segundo lugar, el fracaso de las políticas de promoción de la producción agropecuaria, como ha sucedido en Argelia. Observemos de cerca los dos casos más importantes.

La agricultura martoquí es, sin lugar a dudas, la más potente del Magreb. Su historia colonial (Swearingen, 1988) presenta una primera etapa, que se extiende desde mediados del siglo XIX a principios de los años treinta, en la cual se fomentó la cerealicultura. Las autoridades francesas, sobretodo desde el establecimiento del Protectorado en 1912, pretendieron transformar Marruecos en una despensa de la metrópoli. Ello se explica por la penuria de grano que vivió Francia después de la Primera Guerra Mundial, la vigencia del mito de Marruecos como el granero del Imperio romano y la baja inversión que exigía la producción de cereales. Se constituyeron grandes haciendas en manos de colonos y, también, muchos agticultores nativos desplazaron sus tierras hacia el cultivo del trigo. La rentabilidad estaba asegurada por los altos precios pagados por la metrópoli. Pero, con el inicio de los años treina, el aumento progresivo de la producción interior francesa, así como la irregularidad de las cosechas en Marruecos, cosa que entorpecía la estabilización del mercado, sen- 
Tabla 3. Superficie agraria del Magreb (en miles de hectáreas)

\begin{tabular}{llccc}
\hline & Cultivada & \% del total & Regadío & $\%$ cultivada \\
\cline { 2 - 5 } Argelia & 7.200 & 3 & 300 & 4,1 \\
Marruecos & 8.061 & 11,3 & 1.100 & 13,6 \\
Túnez & 4.900 & 29 & 200 & 4,1 \\
\hline
\end{tabular}

taron las bases para un cambio de política agraria. Comenzó así una segunda fase de la agricultura marroqui, la cual es todavia vigente: la expansión de las tierras de regadío dedicadas al cultivo de productos hortofrutícolas (cítricos y tomates, preferentemente) que, dado su carácter de tempranos respecto de los europeos, son destinados a la exportación (Guerraoui, 1986). La rentabilidad de esta agricultura explica porque las tierras de más calidad han sido acaparadas por los notables del país: actualmente existirían poco menos de 9.000 propietarios que, con un total de 2,2 millones de hectáreas, dispondrían de unas 525 mil hectáreas susceptibles de irrigación adecuada. Esta oligarquia terrateniente, ya existente durante la etapa colonial, amplió sus haciendas comprando los 1,3 millones de hectáreas propiedad de europeos a finales del Protectorado. Posteriormente, ha ido adquiriendo tierras de secano de procedencia diversa que se han revalorizado con la constitución de perímetros de regadío. Acaparó, también las 245 mil hectáreas que, siendo de propiedad pública desde 1973, fueron privatizadas a mediados de los ochenta.

Junto con esta agricultura avanzada sobreviven los campesinos tradicionales: al menos un millón y medio de pequeñas y medianas explotaciones de hasta 50 hectáreas, ocupando un total de más de 6 millones de hectáreas, de las cuales $275 \mathrm{mil}$ son irrigables en diverso grado ${ }^{12}$. No siempre son tierras en propiedad, ya que muchas parcelas se cultivan bajo condiciones de khamasa ${ }^{13}$. Finalmente, el sector más precario del campesinado lo constituye las más de 500 mil familias de agricultores sin tierra.

La naturaleza política del régimen cherifiano hace poco probable una profunda reforma agraria que reclistribuya la tierra de los latifundios, especialmente los de regadío. Además, la baja presión fiscal que éstos soportan, explicable básicamente por motivos políticos ya que se trata de una actividad de enclave fácil de fiscalizar, imposibilita que parte de los recursos generados por la agricultura de exportación sean reconducidos hacia la importación de cereales, la producción interna de los cuales es deficitaria. Por otra parte, las inversiones agra-

12. Este colectivo incluye los beneficiarios de lotes de tierra provenientes de las $372 \mathrm{mil} \mathrm{hec-}$ táreas que, entre 1956 y 1986, fueron distribuidas en varias reformas agrarias (Swearingen, 1988).

13. Se trata de una forma de parceria que supone la siguiente partición de la producción bruta: una quinta parte la retiene el carnpesino (khammas) en compensación por el trabajo dedicado al cultivo, y el resro queda en manos del propieratio (fellab) por haber aportado la semilla y haber asegurado el sostenimiento de los animales de tiro, así como en concepto de cánon por el uso de la finca (Griffin, 1976). 
rias se han concentrado en la extensión de la superficie de riego, dejando a un lado la mejora de los rendimientos de los cereales en el secano. En efecto, entre 1956 y 1986 hasta 29 embalses, construidos o proyectados, se han añadido a los 14 existentes antes de la independencia ${ }^{1 / 4}$.

Argelia fue colonia de poblamiento durante la ocupación francesa. Así, en el momento de la independencia (1962) más de un millón de europeos vivían en el país y poseían unos 2,7 millones de hectáreas (Coté, 1976). Esta agricultura se dedicaba a la exportación, bajo condiciones preferenciales, de vino, cítricos y cereales hacia la metrópoli. Mientras tanto, la población autóctona fue desplazada de las llanuras hacia las zonas montañosas. Ello dió lugar al desbroze de áreas forestales con el fin de obtener tierras de cultivo siendo, no obstante, de poca calidad y muy expuestas a la erosión. Adernás, dada la importancia de la ganadería para la autosubsistencia de los campesinos, el pastoreo llegó a ser abusivo. Ambos factores, así como la destrucción sistemática de los bosques durante la guerra de la independencia y los incendios, han contribuido a la desaparición de las masas forestales argelinas ${ }^{15}$.

Después de la independencia las fincas de los colonos se convirtieron en unas 3000 grandes explotaciones, con un total de 117 mil hectáreas de regadío. A finales de los años ochenta, estas explotaciones estatales, formalmente en régimen de autogestión, fueron divididas en bloques de 80 hectáreas y repartidas entre 7 o 8 familias de ex-asalariados (Aït Amara, 1990).

Por otra parte, con los objetivos de mejorar las condiciones de vida de parte del medio millón de campesinos con poca o sin tierra, ampliar la superficie cultivada y fijar la población rural, en 1971 se puso en marcha la Revolución agraria argelina (Ollivier, 1976). Se expropiaron un total de 1,1 millones de hectáreas provenientes, mayoritariamente, de tierras comunales las cuales fueron redistribuidas entre 85 mil familias.

Visto en perspectiva temporal, el sector agrario argelino no ha contribuido a la reducción de la dependencia alimentaria del país ni tampoco ha sabido actuar como freno del éxodo rural. De forma sucinta, las principales razones que explican este fracaso son:

1. Un importante handicap fue la falta absoluta de personal cualificado en agronomía y en gestión de las explotaciones heredadas de la época colonial. Éstas, además, estaban en pésimo estado de conservación ${ }^{16}$. No resul-

14. La construcción de la infraestructura de riego ha concentrado el $70 \%$ del esfuerzo inversor público en la agricultura entre 1965 y 1986 (Swearingen, 1987).

15. En efecto, se estiman en más de 4,5 millones las hectáreas de bosque existentes en 1830 que, reducidas a un máximo de 2,5 millones, al gunas en avanzado estado de deterioro, allá por 1962 apenas alcanzaban las 500 mil a mediados de los ochenta (Temmar, 1983).

16. Por ejemplo, un $84 \%$ de las instalaciones de los 12 grandes pertmetros de regadío del paśs - con un total de 63,2 mil hectáreas- eran anteriores a la Segunda Guerra Mundial y, además, el $75 \%$ de la superficie total presentaba problemas de satinización. Por otro lado, en el momento de la independencia, más de la mitad de los viñedos tenían más de 25 años (Temmar, 1983). 
ta, pues, sorprendente que los rendimientos de las grandes explotaciones fuesen al principio muy insatisfactorios. Más tarde, el subsector se convirtió en un fuerte consumidor de subvenciones: la renta petrolera alimentó unas retribuciones de los factores productivos sin correspondencia con su eficacia, la cual tampoco mejoró con el paso de los años.

2. Los canales cooperativos para suministrar recursos a las explotaciones mostraron muchas deficiencias: macrocefalia administrativa, diseño de planes de producción con objetivos contradictorios o inalcanzables, retrasos en la llegada de los inputs, pésima infraestructura de almacenaje, falta de un servicio de reparaciones cualificado y suficientemente provisto de piezas de recambio, etc.

3. Los recursos destinados a la modernización del sector agrario no se correspondieron con los disponibles gracias al boom petrolero, debido a la prioridad otorgada a la industria (Goumeziane, 1994). A mediados de los setenta sólo un $15 \%$ de las inversiones se dirigían a la agricultura, sector donde había excesivas diferencias entre la inversión prevista y la realizada.

La debilidad de la agricultura magrebí ha coincidido con un fuerte aumento de la población: los tres estados albergaban unos 35 millones de almas en 1970 , siendo unas 60 en 1990. La tasa de crecimiento de la población de Argelia ha sido del $3 \%$ anual, ligeramente por debajo se ha situado Marruecos, mientras que Túnez ha oscilado alrededor del $2 \%$. A finales de los ochenta se ha manifestado una cierta aceleración motivada por la llegada a la edad fértil de las numerosas generaciones nacidas los años sesenta y setenta, a pesar de que el número medio de nacimientos por mujer ha descendido desde los seis y más hijos a mediados de los setenta, a menos de cinco a finales de los ochenta (Escallier, 1991).

La combinación de los problemas de la agricultura y la evolución demográfica ha supuesto:

1. La aceleración del éxodo rural de los campesinos. Si bien el fenómeno de la emigración del campo hacia las zonas urbanas data de la época colonial, las tres últimas décadas la población urbana ha crecido a una media del 5\% anual (Aür Amara, 1990). Las áreas de mayor atracción han sido las Ilanuras litorales de Argel, Orán y Annaba en Argelia, así como el eje urbano Casablanca-Rabat-Kénitra en Marruecos. Durante los años setenta, atraídas por las mayores retribuciones percibidas por los asalariados de las nuevas industrias dedicadas a la transformación de los recursos minerales y energéticos, las ciudades magrebíes recibieron hasta $300 \mathrm{mil}$ emigrantes al año. No obstante, la mayor parte de tales inmigantes pasaron a engrosar las filas de la economía informal urbana. Este flujo migratorio ha convertido la falta de vivienda y de infraestructura urbana (agua, electricidad, alcantarillado, etc.) en un grave problema socioeconómico.

2. El aumento de las compras exteriores de alimentos básicos: mientras que, a principios de los sesenta, la media anual de importaciones de cereales era 
de unas 300 mil toneladas, durante los ochenta se superaron los 2 millones de toneladas. Unas compras que representaban la mitad o más de la producción interna de grano. A su vez, el Magreb también ha importado azúcar, aceites comestibles, etc. (Calatrava \& Lorca, 1990). La peor situación es la de Argelia. El crecimiento de las compras exteriores, entre éstas las de alimentos, en respuesta a los mayores ingresos obtenidos con las alzas del precio de los hidrocarburos, así como la expansión demográfica y urbana superando la capacidad productiva de la propia agricultura, han hecho que la tasa de cobertura de la balanza agroalimentaria pasase del $58 \%$ en el año 1971 a poco menos del $1 \%$ en 1989. De hecho, desde 1978 se ha situado por debajo del $10 \%$.

\section{La deuda externa y las políticas de ajuste de los años ochenta}

La mayor parte de los recursos financieros exteriores llegados al Magreb han sido prestados, de forma que la deuda externa es un elemento que caracteriza sus economías ${ }^{17}$. La tabla expuesta a continuación muestra, para el año 1992, el volumen de la deuda acumulada y la cuantía de su servicio (esto es, pagos por intereses más amortizaciones de capital) en millones de \$, así como algunos ratios significativos (tabla 4$)^{18}$.

Las variables de la proporción de la deuda externa sobre el PIB y el porcentaje de su servicio sobre los ingresos exteriores (esto es, los ingresos por exportaciones y el saldo de las remesas de los emigrantes y del turismo) no han hecho más que crecer desde principios de los añossetenta.

Por su parte, la evolución de las transferencias netas tampoco ha sido nada favorable. Es decir, la diferencia entre las entradas anuales de nuevos créditos menos el servicio de la deuda, o también la magnitud de los fondos realmente disponibles, ha sido negativa desde 1981, alcanzando un total acumulado de 17,9 mil millones de $\$$ hasta 1992.

La deuda presenta un cuadro particular para cada país. Durante los años setenta el perfil de la deuda exterior argelina fue creciente hasta alcanzar los 19.300 millones de $\$$, en consonancia con la fuerte intensidad inversora del pais $^{19}$. Pero, entre 1981 y 1984 , el volumen descendió por debajo de los 16

17. Las inversiones directas o en cartera procedentes del resto del mundo han sido escasas: entre 1975 y 1990 , las inversiones de capital exterior se han estimado en un rotal inferior a los 5 mil millones de $\$$. Su origen ha sido la CEE/UE y los estados del Golfo Pérsico. En cambio, los fondos prestados han sido mucho más importantes: entre 1978 y 1990 , la Comunidad a cravés de los protocolos, los acuerdos de Lomé y el BEI ha facilitado unos 2,5 mil millones de $\$$, sts estados miembros pueden haber otorgado otros $12 \mathrm{mil}$ millones más, sobretodo en forma de créditos comerciales y, finaimente, desde el Machuek se ha prestado una cifra similar (véanse Khader, 1992; Sid Ahmed, 1987; Ryck, 1987 y Gazzo, 1987 y 1988).

18. Información extraída de fuentes estadisticas internacionales y ejaboradas en Mir, 1993.

19. En 1973 la deuda representaba el $36 \%$ del PIB y su servicio el $13 \%$ de las exportaciones. En 1979 , las cifras fueron del 46 y $27 \%$, respectivamente. Debe de tenerse en cuenta, tam- 
Tabla 4. El peso de la deuda externa en 1992

\begin{tabular}{|c|c|c|c|c|c|c|}
\hline & Deuda & Servicio & $\frac{\text { Deuda }}{\text { PIB }}$ & $\frac{\text { Servicios }}{\text { Ingrs. Ext. }}$ & $\frac{\text { Deuda }}{\text { cáp. }}$ & $\frac{\text { PIB }}{\text { cáp. }}$ \\
\hline & $\overline{26.34}$ & 9.276 & $* 60 \%$ & ${ }^{*} 68 \%$ & $1.000 \$$ & $1.680 \$$ \\
\hline Marruecos & 21.418 & 2.072 & $74 \%$ & $27 \%$ & $840 \$$ & $1.125 \$$ \\
\hline Túnez & 8.476 & 1.377 & $55 \%$ & $24 \%$ & $1.000 \$$ & $1.850 \$$ \\
\hline
\end{tabular}

* Dazos correspondientes a 1991

mil millones de $\$$ (un $31 \%$ del PIB). Se aprovechó, pues, la buena coyuntura provocada por el segundo boom petrolero para cancelar préstamos exteriores. No obstante, el desplome de los precios del crudo a mediados de los ochenta exigió solicitar nuevos para poder sostener el ritmo de expansión de la economía y las compras de alimentos.

En el caso de Marruecos, la deuda externa presentó una rápida expansión a lo largo de la segunda mitad de los años setenta, motivada por la mejora de las expectativas económicas del gobierno a raíz del aumento de los precios del fosfato. Su descenso después de 1976 fue considerado transitorio, de forma que las autoridades, fervientemente convencidas de disponer de una materia prima con un mercado solvente, no dudaron en endeudarse del exterior, asignando los fondos hacia la construcción de industrias (químicas), la expansión de los servicios de la administración, las compras de material militar, etc., todo ello dentro de una reforma al alza del Plan quinquenal 1973-1977 (El Malki, 1989). La persistente atonía de las exportaciones, la carga de la factura petrolera y del servicio de la deuda, así como la amplitud del déficit presupuestario, dieron paso a medidas de ajuste económico tras 1981. Entre 1980 y 1985 la deuda acumulada llegó a un espectacular 1.29\% del PIB, mientras que su servicio se aproximaba al $40 \%$ de todos los ingresos exteriores. Posteriormente, el peso de la deuda tendió a la baja reflejando, en buena parte, el efecto de los sucesivos acuerdos para el aplazamiento temporal de las devoluciones de capital.

Entre 1977 y 1980, con la confianza que daba a Túnez la exportación de crudo y de fosfatos, la deuda creció rápidamente. Durante la primera mitad de los años ochenta la expansión de la deuda continuó a un ritmo más moderado. Las medidas de ajuste frenaron una trayectoria que, en 1987, la había situado en el $71 \%$ del PIB. Por su parte, desde 1984, el ratio servicio/entradas exteriores ha oscilado entre el $20 \%$ y el $30 \%$.

La carga excesiva de la deuda llevó a los estados magrebíes a aplicar políticas de ajuste económico (Bourenane, 1991; Rhazaoui, 1987; Mahjoub, 1989). Dos han sido las líneas de actuación: la reducción del déficit presupuestario y la incidencia sobre los saldos exteriores. En el primer capitulo se incluyen el

bién, que las autoridades argelinas suscribieron la mayor parte de los crédiros con entidades financieras privadas a tipos de interés flotante y con plazos de amortización relativamente cortos (Mir, 1993). 
freno a la expansión del sector público, acompañado del saneamiento y la venta de empresas estatales ${ }^{20}$. Otra medida de importante repercusión social ha sido la reducción de las subvenciones a los bienes alimenticios básicos. Esta decisión ha generado diversos levantamientos populares ${ }^{21}$. Con respecto al sector exterior, la política de ajuste ha intentado mejorar el déficit comercial a través de la devaluación monetaria, el freno a las importaciones de bienes de capital $y$ de dar facilidades a las inversiones extranjeras en empresas off-shore.

\section{El turismo y las remesas de los emigrantes}

Este apartado debe de empezar haciendo una breve referencia a los saldos comerciales de los paises del Magreb. En el caso de Argelia a menudo el saldo fue positivo, siendo más elevado cuanto más aito era el precio de los hidrocarburos. Efectivamente, el superávit comercial equivalió al $8 \%$ del PIB en 1982 frente al 1,7\% el 1988. Marruecos y Túnez, por su parte, han necesitado otras fuentes de ingresos exteriores para compensar sus déficits comerciales crónicos que, en ocasiones superaron el 10\% del PIB. Tales ingresos extras han procedido del turismo y de las transferencias de los emigrantes.

El turismo en Marruecos pasó de los 370 mil visitantes en 1964 a más de un millón doscientos mil en 1973. Luego, la expansión se estancó. No obstante, desde mediados de los ochenta el flujo de turistas volvió a crecer, alcanzando los cuatro millones y medio en 1992 (Sebbar, 1994). En el caso de Túnez, el número de visitantes también creció rápidamente: de $750 \mathrm{mil}$ en 1972 a 3,45 millones en 1989 (Baiget, 1991). A pesar de que se trata de cifras estimativas, la importancia económica del turismo resulta indiscutible durante la segunda mitad de los años ochenta, los ingresos turísticos representaron el $4 \%$ del PIB marroquí y el doble del tunecino (Mir, 1993). Por lo que se refiere a los ingresos en valores absolutos, en el año 1989 se alcanzaron los 1.015 y 904 millones de $\$$, respectivamente. Una última cifra: recién iniciados los noventa, el turismo capturaba el $10 \%$ de las divisas totales reunidas por Marruecos y el $30 \%$ de las de Túnez.

No obstante, las cifras presentadas no deben de impresionarnos. En primer lugar, es necesario advertir que no todos los visitantes se ajustaron a la defini-

20. Durante la segunda mitad de los setenta y la primera de los ochenta la participación del Estado en la economía creció rápidamente, tanto por el tejido industrial levantado como por la expansión de las instancias administrativas. Ast, por ejemplo, el sector público representaba un mínimo del $60 \%$ de toda la inversión en Marruecos y en Túnez, mientras que el estado argelino creó, entre 1967 y 1987, más de millón y medio de puestos de trabajo (véanse Goumeziane, 1994 y Amrani, 1991).

21. Entre otros cabe destacar los de junio de 1981 en Casablanca, en enero de 1984 en Marraquech y el Rif, y en diciembre de 1990 en Fes y en otras ciudades, por to que se tefiere a Marruecos; en noviembre de 1986 en Constantina y Sétif, y en octubre de 1988 en la capizal, en el caso de Argelia, y en los meses de diciembre-enero de 1983-1984 en Túnez. Debe de recordarse que, durante el periodo 1980-1984, tales subvenciones significaban el 0,9\% del PIB argelino, el 1,9\% del marroquí y el 2,9\% del runecino (Khader, 1992). 
ción de turista como ciudadano extranjero que pasa varios días o semanas en estricto ocio. Por ejemplo, sólo el 36\% de los visitantes de Marruecos en 1992 fueron propiamente turistas (europeos - sobre todo franceses-, americanos y árabes no magrebies). Un $25 \%$ de las visitas las efectuaron marroquies tesidentes en el exterior. El restante $38 \%$ de los presuntos turistas fueron fundamentalmente argelinos en cortos viajes de compras a las localidades fronterizas. En segundo lugar, comparado con el Mediterráneo noroccidental, las cifras anteriormente expuestas son ridículas: El año en cuestión España, Francia e Italia recibieron 54, 50 i 55 millones de visitantes, respectivamente.

La presión para equilibrar lás cuentas exteriores y la probada capacidad de creación de puestos de trabajo por parte del sector turístico explican los esfuerzos de los gobiernos de ambos países en el terreno de la promoción, así como el hecho de otorgar todo tipo de facilidades financieras y fiscales a nuevos operadores turísticos, sean nacionales o extranjeros.

Aunque sea cierto que buena parte del potencial turístico del Magreb esté todavía por explotar, la opción en favor de esta actividad plantea algunos problemas:

1. En primer lugar, es sabido que los flujos de visitantes está muy influenciado por las eventualidades políticas externas ${ }^{22}$. También influyen, especialmente en el caso magrebí, las contingencias internas. Esto es, la presión política de los movimientos islamistas y la forma como ello queda reflejado en la prensa europea. La inestabilidad política de la región es un grave obstáculo para la expansión de la industria turística.

2. En segundo lugar, el turismo supone una fuerte presión sobre los recursos hídricos del Magreb. En efecro, la costumbre de consumir grandes cantidades de agua en sus países de origen, así como la preocupación por la higiene dado que visitan un país pobre, caracterizan la actitud de los turistas centroeturopeos hacia la disponibilidad del preciado Ifquido. Ello explica que en Túnez, por ejemplo, el módulo turístico sea de 715 litros por día y cama, mientras que a los habitantes de la capital se les asigna una cantidad mínima de 150 litros/día (Troin, 1991).

Las transferencias de los trabajadores emigrados juegan un importante papel en las las economías magrebíes, pero su magnitud y signo son muy diferentes según el país. Los más favorecidos son Marruecos y Túnez. A finales de los ochenta se estimaban en unos 750 mil los marroquies y en $300 \mathrm{mil}$ los tunecinos residentes en países de la CEE/UE. Sus remesas entre 1985 y 1989 representaron una media anual del $7 \%$ del PIB marroquf y del $5 \%$ del

22. Así, por ejemplo, en 1986 el ataçue aéreo israelí al cuartel central de la OLP en Túnez, conjuntamente con la incursión norteamericana en Libia, provocaron un descenso del $25 \%$ de los visitantes llegados a Túnez con respecto a los 2 millones de turistas del año anterior. Por contra, el pais resultó favorecido po: el cierre de la frontera entre Libia y Egipto en 1988. 
PIB tunecino ${ }^{23}$. Al margen de haber contribuido a compensar los déficits exteriores de ambas economías, a un nivel más concreto tales recursos han tenido un doble efecto: asegurar la subsistencia de los miembros de familias rurales sin tierra y/o trabajo continuado e incentivar la actividad económica de las localidades donde los fondos tienen su destino final. En efecto, una parte de ellos se destinan a mejoras en el hogar y, en el caso de parejas jóvenes, a la compra del solar y posterior edificación de la futura vivienda familiar. Ello genera la demanda de una amplia variedad de objetos artesanales e industriales, lo cual contribuye decididamente al sostén de la actividad económica de muchas comarcas. Los beneficiosos efectos generales y particulares de las transferencias explica la preocupación con que las autoridades de ambos Estados magrebíes contemplan la integración de las familias de los emigrantes en su país de destino, ya que ello supone el descenso de las remesas.

Argelia contaba en 1990 con más de 845 mil emigrantes en Europa (casi todos en Francia). A pesar del elevado volumen de emigrantes, las transferencias contabilizadas han sido irrisorias. La explicación radica en el hecho que las autoridades argelinas nunca tomaron medidas para captar tales recursos monetarios. La política de fomento de las inversiones industriales para poner en pie la infraestructura necesaria para la extracción, el transporte y la transformación del gas natural y el petróleo crudo, impulsó a los gobiernos a fijar un cambio ofrcial inflado del dinar frente al dólar, ya que así las importaciones eran comparativamente más baratas en términos de la moneda nacional. Adicionalmente, la prioridad dada a las inversiones industriales en el sector de los hidrocarburos frente a la fabricación de artículos de consumo duraderos, provocaron una demanda insatisfecha por parte de los colectivos de la sociedad argelina con mayor poder adquisitivo, especialmente durante los 12 años del boom petrolero.

La primera circunstancia desincentivo los emigrantes a convertir sus ahorros en dinares, mientras que la segunda hizo muy atractivo adquirir todo tipo de pequeños artículos de consumo en el país de destino para poder revenderlos en el mercado negro argelino durante los periodos de vacaciones.

Estas prácticas, más el contrabando cotidiano de productos desde Marruecos y Túnez han constituido el llamado trabendo.

\section{Conclusión}

El diagnóstico general sobre la trayectoria seguida por las economías magrebíes desde los años setenta a los noventa integra los elementos que explican

23. En concreto, durante estos mismos años, en el reino alauita entaron una media anual de 1.319 millones de $\$$ y en Túnez unos 425 millones de $\$$. Ia magnitud de las transferencias no cesb de crecet a lo largo de las tres últimas décadas: expresadas en términos corrientes, en I970 Marruecos ingreso 63 millones de $\$$ por remesas, mientras que en 1992 las entradas fueron 35 veces mayores (Mir, 1993). 
cómo un arranque con unas inmejorables expectativas ha terminado, tras ciertos vaivenes, en una profunda crisis socioeconómica.

El motor que ha impulsado todo el proceso han sido las exportaciones de materias primas energéticas y minerales, los precios de las cuales manifestaron una gran volatilidad. Sus oscilaciones se transmitieron inmediatamente y con gran amplitud a la economía irtterna.

Durante los años del boom los gobiernos otorgaron la máxima prioridad al desarrollo de la industria. Esta expansión se financió con las rentas de los hidrocarburos y el fosfato, así como tomando prestadas grandes sumas de capitales exteriores. Pasando por alto la valoración de la eficacia con que se invertieron tales recursos, tras el cambio de coyuntura en el mercado internacional, el peso del endeudamiento liegó a ser insoportable. La posterior puesta en marcha de políticas de ajuste económico provocó un aumento del paro y de la pobreza, reflejados en el crecimiento de la economía informal urbana y en una mayor presión migratoria hacia el exteriot.

Visto en perspectiva, quizás el coste más oneroso para los países del Magreb procede del olvido de la agricultura. Al no disponer de capacidad para generar excedentes suficientes para alimentar la propia población, ha sido imprescindible el recurso a las importaciones masivas de alimentos. Aunque las cantidades compradas dependan de las cosechas interiores, que oscilan según el azar climático, se trata de una partida muy rígida, ya que juega un papel clave para evitar que empeore el clima sociopolítico del país. Indudablemente, la cooperacion internacional puede jugar un papel decisivo en la consecución del objetivo de elevar los rendimientos de la agricultura de secano y en la mejora de la ganadería, así como también para detener la erosión del suelo mediante la reforestación.

\section{Bibliografía}

ABC (Arab Banking Corporation) (1986). The Arab Economies. Structure and Outlook. Londres: Arab Banking Corporation.

Art AMARA, Hamid (1990). "Pression démographique et dynamique des structures agraires au Maghreb". Mondes en developpement, vol. 18, núm. 71, p. 9-17.

AMRANI, Hassan (1994). «El proceso de privatización». Boletín económico de ICE, núm. 2400-2401, p. 303-307.

BAIGET, Fernando (1991). "Estructura económica de Túnez». Boletín Económico ICE, núm. 2283, p. 1934-1937.

BENOUNE, Mahfoud (1988). The making of contemporary Algeria (1830-1987). Colonial upheabals and Post-independence development. Cambridge: Cambridge University Press.

BOURENANE, Naceur (1991). "La economfa argelina a la hora de las grandes decisiones". Boletín Económico ICE, núm. 2283, p. 1922-1928.

Calatrava, A.; LORCA, A. (1990). "La insuficiencia alimentaria en los países del Magreb: factor clave de endeudamiento". Información Comercial Española, núm. 684-685, p. 159-173. 
CALATRAVA, Ascensión (1991). "El sector textil como motor de las manufacturas». Boletin Económico ICE, núm. 2283, p. 1950-1956.

Chevalier, A; Kessler, V. (1990). "Croissance et insertion internationale du Maghreb. Questions sur l'avenir des relations avec l'Europe", en KODMANI-DARWISH, B. (ed.). Maghreb: les anées de transition. París: IFRI-Masson.

COTE, Marc (1976). "Révolution agraire et sociétés agraires: Le cas de l'est algérien". Annuaire de l'Afrique du Nord 1975, vol. 14, p. 173-184.

DelmaSuRE, Dominique (1990). "L'économie tunisienne: De l'État-providence à l'ambition liberalen. Mondes en Développement, vol. 18, núm. 71, p. 19-33.

EL MALKI, Habib (1989). Trente ans d'économe marocaine 1960-1990. París: CNRS.

ESCALLIER, R. (1991). "Démographie et migrations. Obsession du nombre et considérable rajeunissement de la population", en LACOSTE, C.; LACOSTE, Y. (eds.). L'État du Maghreb. París: I_a Découverte, p. 79-90 y $91-97$.

Gazzo, Yves (1987), "L'endettement du Maghreb au sein du monde arabe", en Michel, H.; SANTUCCI, J.-C. (eds.). Le Maghreb dans le monde arabe ou les affinetés sélectives. París: CNRS, p. 271-283.

- (1988), "Les financements internationaux et les pays du Maghreb", en ClAISSE, A.; CONAC, G. (eds.). Le grand Maghreb. Donées socio-politicas et facteurs d'integration des états du Maghreb. París: Economica, p. 281-292.

GOUMEZIANE, Smail (1994). Le mal algérien. Economie politique d'une transition inachevee 1962-1994. París: Fayard.

Guerraoul, Driss (1986). Agriculture et developpement au Marroc. París: Publisud.

GRIFFIN, Keith (1976). Land Concentration and Rural Poverty. Londres: MacMillan Press.

JORDAN GALDUF, J.M. (I99I). "Agricultura tradicional "versus" agriculrura moderna». Boletín Económico ICE, núm. 2283, p. 1943-1949.

KHADER, Bichara (1992). Europa y el gran Magreb. Barcelona: Fundación Paulino Torras Domènech.

MaHJoub, Azzam (1989), "État, secteur public et privatisation en Tunisie", Anuaire de l'Africa du Nord 1987, vol. 26, p. 298-315.

MIR, Pere (1993). "Economy and the Foreign Sector in the States of the Arab Maghreb Union (the decades of the 1970 s and 1980 s. Working Papers/Papers de recerca. CEPD-Universitat Autònoma de Barcelona, núms. 3 y 4, 119 p.

OLLIVIER, Marc (1976). "Place de la Révolution Agraire dans la stratégie algèriene de développement». Anuaire de l'Africa du Nord 1975, vol. 14, p. 91-114.

OUALALOU, Fathallah (1975). "La politique phospharière marocaine et les tentatives d'adaptation aux vatiations de la demande internationale". Annuaire de l'Afrique du Nord 1974, vol. 13, p. 41-70.

PASCON, Paul (1985). "La petite et moyenne hydraulique au Marroc. Problèmes institutionels et juridiques posés par son extension au Marroc», en CONAC, G.; SAVONnET-GUYot, C.; ConaC, F. (eds.) Les politiques de l'eau en Afrique. Développement agricole et participation paysanne. París: Economica, p. 443-477.

POMFRET, Richatd (1987), "Morocco's international economic relations", en ZARTMAN, I.W. (ed.). The political economy of Morocco. Nueva York: Praeger, p. 173-187.

RHAZAOUI, Ahmed (1987). "Recent economic trends: Managing the indebtedness", en ZARTMAN, I.W. (ed.). The political economy of Morocco. Nueva York: Praeger, p. $141-158$. 
RYCK, Jean-François (1987). "Les rapports d'investissement entre les pays du Golfe et les états arabes d'Afriquen, en MiCHEL, H.; SanruCCI, J.-C. (eds.). Le Maghreb dans le monde arabe ou les affinetés sélectives. París: CNRS, p. 243-270.

SEBBAR, Hassan (1994). «El turismo internacional». Boletín económico ICE, núm. 2400. 2401, p. 359-366.

SÉRENJ, Jean-Pierre (1991). "Les rentes pétrolières et minières", en LACOSTE, C.; Lacosre, X. (eds.). L'Etat du Maghreb. París: La Découverte, p. 469-472.

SID-AHMED, Abdelkader (1987). La place du Maghreb dans les investissements et les flux financiers des états arabes détenteurs de pétro-dollars", en MicHEL, H.; SANTUCCl, J.-C. (eds.). Le Maghreb dans le monde arabe ou les affinetés sllectives. París: CNRS, p. 217-241.

SWEARINGEN, Will D. (1987). "Morocco's agricultural crisis", en ZAR'TMAN, William I. (ed.) The political economy of Morocco. Nueva York: Praeger, p. 159-172.

- (1988). Moroccan Mirages. Agrarian Dreams and Deceptions (1912-1986). Londres: I.B. Tauris \& Co. Ltd.

Temmar, Hamid M. (1983). Strategie de developement independant. Le cas de l'Algérie: un bilan. París: Publisud.

Toumi, Mohsen (1990). "Des développements "Ego-centrés" au développement solidaire", en KODMANI-DARWISH, Bassma (Ed.). Maghreb: Les antes de transition. París: IFRI-Masson, p. 147-187.

TROIN, Jean-François (1991). "Le problème de l'eau, un enjeu stratégique", en LACOSTE, C.; Lacoste, Y. (eds.). L'État du Maghreb. París: La Découverte, p. 457-458.

ZENAIDI KARRAY, Bourane (1987). Le marche mondial des phosphates et des engrais phosphates. París: Economica. 